\title{
Chemical Composition, Antifungal and Antitumor Properties of Ether Extracts of Scapania verrucosa Heeg. and its Endophytic Fungus Chaetomium fusiforme
}

\author{
Lei Guo ${ }^{1}$, Jin-zhong Wu ${ }^{1,2}$, Ting Han ${ }^{1}$, Tong Cao ${ }^{3}$, Khalid Rahman ${ }^{4}$ and Lu-ping Qin ${ }^{1, *}$
}

1 Department of Pharmacognosy, School of Pharmacy, Second Military Medical University, Shanghai 200433, P.R. China

2 Academy of integrative medicine, Fujian University of Traditional Chinese Medicine, Fuzhou, Fujian 350003, P.R. China; E-mail: jinzhongfj@126.com

3 Department of Biology, College of Life and Environment Sciences, Shanghai Normal University, Shanghai 200234, P.R. China; E-mail: CT1946@263.net

4 Faculty of Science, School of Biomolecular Sciences, Liverpool John Moores University, Byrom Street, Liverpool L3 3AF, England, UK

* Author to whom correspondence should be addressed; E-mail: qinsmmu@126.com; lpqin@smmu.edu.cn.

Received: 15 June 2008; in revised form: 19 August 2008 / Accepted: 3 September 2008 / Published: 4 September 2008

\begin{abstract}
An endophytic fungus Chaetomium fusiforme was obtained from a liverwort, Scapania verrucosa. A comparison of the constituents of the ether extracts between $S$. verrucosa and the $C$. fusiforme culture was investigated by gas chromatography-mass spectrometry (GC/MS). The yield of ether extract based on dried plant material was $0.6 \%$ and 59 compounds were found in S. verrucosa. $(+)$-Aromadendrene (9.12\%), hexadecanoic acid (6.92\%), 6-isopropenyl-4,8a-dimethyl-1,2,3,5,6,7,8,8a-octahydro-naphthalen-2-ol (5.97\%), s-tetrachloroethane (5.61\%) and acetic acid (5.30\%) were found to be the most abundant components among the 49 characterized compounds in S. verrucosa, which represented $84.64 \%$ of the total extract. However, the constituents of the cultured endophyte extract contained mainly acetic acid (35.05\%), valeric acid, 3-methyl-, methyl ester (21.25\%), and butane-2, 3-diol (12.24\%). Although the extracts of $S$. verrucosa and its endophyte showed little chemical composition correlation, both of them demonstrated antifungal and antitumor activities. Furthermore, $C$. fusiforme has displayed a wider range
\end{abstract}


of antimicrobial and antitumor activities, which were better than the host plant. These results could support the suggestion of endophytes as an alternative of the host for medicinal activity.

Keywords: Scapania verrucosa; Chaetomium fusiforme; GC/MS; antifungal activity; antitumor activity.

\section{Introduction}

Bryophytes are known to possess various rare and novel natural products. Many of these exhibit interesting biological activities such as antimicrobial, cytotoxic, insect antifeedant, muscle relaxing, some enzyme inhibitory, apoptosis inducing activity, lipoxygenase, calmodulin, hyaluronidase, cyclooxygenase, thrombin inhibitory activity and neuritic sprouting activity [1-5]. Liverworts contain oil bodies which are composed of a large amount of mono-, sesqui- and diterpenoids, as well as aromatic compounds [6, 7]. In particular, liverworts contain pinguisane-type sesquiterpenoids, sacculatane-type diterpenoids and bis (bi-benzyl) aromatic compounds which have not been found in higher plants [2-8].

Scapania verrucosa Heeg. (Scapaniaceae), is a liverwort, mainly found distributed in south-central China, Nepal and the Himalayan region of Jammu and Kashmir. It is about 10-25 mm tall and grows on forest ground, rocks and decaying wood [9, 35]. The plants from Scapaniaceae, such as $S$. aequiloba, S. ampliata, S. aspera, S. bolandeli, S. nemorea, S. ornithopodiodes, S. paludosa, S. parvitexta, S. stephanii, S. subalpina, S. uliginosa, and $S$. undulate were chemically investigated previously [7, 10-15].

Endophyte, by definition, is one which resides in the tissues beneath the epidermal cell layers and causes no apparent harm to the host [16]. Recent studies have shown that fungal endophytes are ubiquitous in plant species $[17,18]$ and are mutualistic to their host. At least some of them are thought to be receiving nutrition from the plant in exchange for producing special substances such as secondary metabolites to protect the host from successful attack by fungi, pests and mammals. In support of this idea, metabolites of endophytes have been reported to inhibit a number of micro-organisms [19, 20]. On one hand, endophytes can produce similar or the same biologically active constituents as its host, such as an endophytic fungus producing taxol [21]. On the other hand, fungi are a prolific source of metabolites with significant biological activities. Many important anticancer, antifungal and antibacterial chemotherapeutics are either microbial metabolites or their semisynthetic derivatives. Investigating the metabolites of endophytic fungi can increase the chance of finding novel compounds. An intensifying stream of attention is being directed to the endophytes from unique habitats living in special environments [22, 23].

The difficulty of acquiring large amounts of liverworts is due to the fact that many of them are spread over wide areas and occur only in small populations. A promising way out of this impasse is the use of endophytes of liverworts. The endophytes could be utilized for their fermentation and biotechnology capabilities as an alternative mode of production of the bioactive components. 
Distinctly from plants, endophytes can be cultured quickly and the biomass can be accumulated by large scale fermentation.

To the best of our knowledge, there are no reports on $S$. verrucosa extracts with antimicrobial or antitumor activity. In this paper, a comparison of the chemical components between $S$. verrucosa and the culture of its endophyte was carried out. This study also reported the antimicrobial and antitumor activities of the essential oil from $S$. verrucosa and its endophyte Chaetomium fusiforme.

\section{Results and Discussion}

\section{Chemical components of the plant and the endophyte extracts}

The yield of pale green colored ether extract of $S$. verrucosa was about $0.6 \%$. The endophyte oil was yellow one. The components identified from $S$. verrucosa extract, their retention indexes and their percentage composition are summarized in Table 1. The results of the GC/MS analysis of the composition of the endophytic fungus and blank ferment broth PDB are presented in Table 2.

Table 1. Chemical composition of $S$. verrucosa extract.

\begin{tabular}{|c|c|c|}
\hline Compounds & $\mathbf{R I}^{\mathbf{a}}$ & Content $/ \%$ \\
\hline n-dodecane & 1200 & 0.22 \\
\hline 2-heptenal & 1334 & 0.18 \\
\hline 3-octanol, acetate & 1344 & 0.19 \\
\hline octen-1-ol, acetate & 1385 & 2.84 \\
\hline n-tetradecane & 1400 & 0.29 \\
\hline acetic acid & 1425 & 5.30 \\
\hline aminic acid & 1470 & 0.87 \\
\hline s-tetrachloroethane & 1516 & 5.61 \\
\hline$\beta$-bourbonene & 1528 & 1.83 \\
\hline aromadendrene, dehydro- & 1541 & 0.42 \\
\hline (-)-aristolene & 1582 & 2.06 \\
\hline$\beta$-elemen & 1599 & 1.30 \\
\hline calarene & 1604 & 5.01 \\
\hline cedr-8(15)-ene & 1609 & 1.05 \\
\hline 1H-cyclopropa[a]naphthalene, decahydro-1,1,3a-trimethyl-7-methylene & 1614 & 2.74 \\
\hline 9-methyltetracyclo[7.3.1.0(2.7).1(7.11)]tetradecane & 1655 & 4.49 \\
\hline$(+)$-aromadendrene & 1660 & 9.12 \\
\hline$\beta$-farnesene & 1672 & 0.51 \\
\hline acroadiene & 1689 & 0.34 \\
\hline n-heptadecane & 1700 & 0.26 \\
\hline thujopsene-(12) & 1715 & 0.19 \\
\hline eremophila-1(10), 11-diene & 1732 & 0.14 \\
\hline c-neoclovene & 1747 & 0.12 \\
\hline
\end{tabular}


Table 1. Cont.

\begin{tabular}{|c|c|c|}
\hline 11-isopropylidenetricyclo[4.3.1.1(2,5)]undec-3-en-10-one & 1784 & 0.09 \\
\hline n-octadecane & 1800 & 0.18 \\
\hline (E,E)-2,4-decadienal & 1821 & 0.16 \\
\hline$(+)$-cuparene & 1838 & 0.13 \\
\hline hexanoic acid & 1851 & 0.15 \\
\hline [2,4,4-trimethyl-1-(2-methylpropanoyloxy)pentan-3-yl] 2-methylpropanoate & 1884 & 0.17 \\
\hline phytol & 1929 & 1.76 \\
\hline cyclohexene, 6-(2-butenyl)-1,5,5-trimethyl-(E)- & 2027 & 0.47 \\
\hline ledol & 2095 & 0.19 \\
\hline 2-(4a,8-dimethyl-1,2,3,4,4a,5,6,7-octahydro-naphthalen-2-yl)-prop-2-en-1-ol & 2124 & 0.67 \\
\hline spathulenol & 2135 & 4.74 \\
\hline guaia-1(5),11-diene & 2162 & 0.09 \\
\hline thujopsene & 2182 & 0.37 \\
\hline 6-isopropenyl-4,8a-dimethyl-1,2,3,5,6,7,8,8a-octahydro-naphthalen-2-ol & 2241 & 5.97 \\
\hline aromadendrene oxide-(2) & 2299 & 1.22 \\
\hline cyclohexene, 6-(2-butenylidene)-1,5,5-trimethyl-(Z,E)- & 2323 & 0.47 \\
\hline (-)-spathulenol & 2464 & 0.41 \\
\hline 7-acetyl-2-methyl-5-isopropylbicyclo[4.3.0]nonane & 2524 & 1.61 \\
\hline thunbergol & 2538 & 0.65 \\
\hline tetradecanoic acid & 2585 & 0.25 \\
\hline platambin & 2686 & 2.22 \\
\hline hexadecanoic acid & 2756 & 6.92 \\
\hline 4,8,13-duvatriene-1,3-diol & 2955 & 3.72 \\
\hline stearic acid & 3020 & 1.82 \\
\hline 9-octadecenoic acid (Z)- & 3072 & 1.24 \\
\hline linoleic acid & 3170 & 3.92 \\
\hline Total identified & & 84.64 \\
\hline
\end{tabular}

${ }^{a}$ calculated from retention times relative to n-alkanes under the same GC conditions (DB-Wax column).

Table 2. A comparative analysis of the composition of Chaetomium fusiforme and the ferment broth PDB without inoculation.

\begin{tabular}{|c|c|c|c|c|c|}
\hline Chaetomium fusiforme & & & & & \\
\hline Compounds & $\mathbf{R} \mathbf{I}^{\mathbf{b}}$ & Content/\% & Compounds & $\mathbf{R I}^{\mathbf{b}}$ & Content $/ \%$ \\
\hline butyl alcohol & 1195 & 1.35 & 2-n-pentylfuran & 1259 & 0.24 \\
\hline methyl 2-oxopropionate & 1276 & 0.14 & ethyl pyroracemate & 1303 & 0.15 \\
\hline acetoin & 1314 & 1.70 & devoton & 1347 & 0.90 \\
\hline 1,2-propanediol & 1336 & 0.21 & ethyl vinyl ketone & 1414 & 0.51 \\
\hline 3-octanol & 1384 & 1.61 & acetic formic anhydride & 1436 & 10.45 \\
\hline acetic acid & 1425 & 35.05 & 3-furancarboxaldehyde & 1455 & 1.29 \\
\hline
\end{tabular}


Table 2. Cont.

\begin{tabular}{|c|c|c|c|c|c|}
\hline 2-furancarboxaldehyde & 1443 & 0.28 & aminic acid & 1470 & 2.39 \\
\hline aminic acid & 1470 & 0.56 & propionic acid & 1493 & 0.36 \\
\hline butane-2,3-diol & 1485 & 11.24 & 2-butyl-1-octanol & 1528 & 0.54 \\
\hline dimethylsuccinate & 1526 & 0.61 & 2-cyclopentene-1,4-dione & 1535 & 0.25 \\
\hline butyrolactone & 1561 & 0.23 & acrylic acid & 1560 & 0.28 \\
\hline acetophenone & 1573 & 0.26 & furfuryl alcohol & 1575 & 2.66 \\
\hline \multirow[t]{2}{*}{ 4-penten-1-ol,2,2,4-trimethyl- } & 1592 & 0.38 & 2-furancarboxylic acid, tetrahydro & 1581 & 0.67 \\
\hline & & & -3-methyl-5-oxo- & & \\
\hline 5-hexalactone & 1669 & 5.71 & n-heptanoic acid & 1623 & 0.27 \\
\hline valeric acid,3-methyl-,methyl ester & 1677 & 21.25 & 2-ethylcyclohexanone & 1651 & 0.57 \\
\hline ethyl 3-hydroxy-2,2-dimethylbutanoate & 1699 & 0.29 & heptadecane,2,6,10,15-tetramethyl- & 1660 & 0.86 \\
\hline benzeneethanol & 1734 & 6.17 & n-caproic acid & 1690 & 1.73 \\
\hline hydroxymethylfurfurole & 1812 & 1.00 & phenol, p-sec-butyl- & 1701 & 0.44 \\
\hline p-cresol & 1835 & 0.25 & 1,2-dimethylpropyl acetate & 1713 & 0.69 \\
\hline o-cresol & 1840 & 0.18 & benzenemethanol & 1717 & 0.24 \\
\hline methyl vinylcarbinol & 1861 & 0.67 & corylon & 1768 & 0.30 \\
\hline benzeneacetic acid & 1878 & 0.26 & maltol & 1777 & 2.47 \\
\hline methyl vinylcarbinol & 1892 & 0.56 & methyl 2-furoate & 1808 & 0.86 \\
\hline methyl phenylglycalate & 1923 & 0.26 & hydroxymethylfurfurole & 1812 & 20.79 \\
\hline monomethyl succinate & 1988 & 2.66 & pyrrole-2-carboxaldehyde & 1814 & 1.26 \\
\hline 2-furoic acid & 2048 & 0.43 & pantolactone & 1818 & 0.76 \\
\hline pyrrolidine-5-one, 2-[3 -hydroxypropyl]- & 2167 & 0.25 & benzeneacetic acid & 1878 & 5.68 \\
\hline 4-(2-hydroxyethyl)phenol & 2565 & 2.37 & 2-methoxy-4-vinylphenol & 1915 & 0.69 \\
\hline \multirow[t]{11}{*}{ total } & & 95.94 & pyranone & 1962 & 8.35 \\
\hline & & & 3,5-dihydroxy-2-methyl-4-pyrone & 1969 & 1.08 \\
\hline & & & hemineurine & 1980 & 2.62 \\
\hline & & & 3-hydroxypyridine & 2048 & 3.11 \\
\hline & & & o-aminophenol & 2054 & 2.50 \\
\hline & & & butanimide & 2081 & 1.96 \\
\hline & & & hexadecanoic acid & 2476 & 1.05 \\
\hline & & & niacinamide & 2549 & 2.61 \\
\hline & & & palmitamide & 2858 & 1.89 \\
\hline & & & cyclo(leucyloproly) & 2870 & 7.81 \\
\hline & & & total & & 91.27 \\
\hline
\end{tabular}

${ }^{\text {a }}$ Potato Dextrose Broth

${ }^{\mathrm{b}}$ calculated from retention times relative to n-alkanes under the same GC conditions (DB-Wax column).

Fifty-nine components were detected in the ether extract of $S$. verrucosa, of which 49 components representing $84.64 \%$ were identified. According to our results, the main constituents of the extract 
from $S$. verrucosa were aromadendrene (9.12\%), hexadecanoic acid (6.92\%), 6-isopropenyl-4,8adimethyl-1,2,3,5,6,7,8,8a-octahydronaphthalen-2-ol (5.97), s-tetrachloroethane (5.61\%), and acetic acid $(5.30 \%)$. Finally, there were ten other volatile chemical constituents that remained unidentified since spectral data obtained did not appropriately match any compound in the NIST database.

The genus Scapania produces many kinds of sesquiterpenoids and diterpenoids which are ubiquitous in other liverworts. The most common sesquiterpenes are anastreptene and aromadendrane hydrocarbons. The European $S$. undulata mainly comprises longifolene-type, longiborneoltype, $(+)-$ ent-epi-cubenol-type, and labdane-type sesquiterpene [11]. S. undulata collected in Belgian elaborates muurolane-type sesquiterpenoids [12, 24]. The major components of the Japanese S. undulata are (-)longiborneol and a-longipinene. In addition, it contains longipinanol, labdanes and dimeric labdanes [10]. The predominant components of S. nemorea in France and Germany are cis-clerodanes and a secoclerodane-type diterpenoid [14]. Another German specimen produces diplophyllolide and a secoclerodane [25-26]. S. bolanderi elaborates not only verrucosane and neoverrucosane diterpenoids [27] but also trans-clerodane-type diterpenoid [13]. S. subalpina and S. uliginosa both elaborate the same sesquiterpene hydrocarbons, longifolene, and isolongifolene. In sum, aromadendranes, clerodanes, labdanes, longibornades, longifolanes, longipinanes, and muurolanes are very valuable chemical markers of the genus Scapania [28]. Comparison with previous study of the chemical composition of related species, $S$. verrucosa also produces a great amount of aromadendrane. However, our investigation showed different qualitative profiles. Calarene, maaliane-type and aristolane-type sesquiterpene, which appear in our study as abundant constituents, seem to be in traces or absent in other species of the genus Scapania.

In the analysis of the ether extract obtained from the culture of Chaetomium fusiforme, there are 40 compounds were found. The number of identified compounds was 28 , which represented $95.94 \%$, while the number in blank broth PDB is 38, representing 91.27\%. As for C. fusiforme, acetic acid (35.05\%), valeric acid, 3-methyl-, methyl ester (21.25\%) and butane-2,3-diol (12.24\%) were characterized as the dominating compounds. In the extract of blank culture medium, hydroxymethylfurfurole $(20.79 \%)$, acetic formic anhydride $(10.45 \%)$, pyranone $(8.35 \%)$, cyclo (leucyloproly) (7.81\%), and benzeneacetic acid (5.68\%) are the most abundant.

It is apparent from the data shown that the components of $S$. verrucosa were predominantly monoand sesquiterpenes, dominated by aromadendrene, whilst its endophytic fungus $C$. fusiforme seldom produced sesquiterpenes. Interestingly, both of them produced not quite abundant acetic acid and aminic acid, which were known to kill a wide spectrum of fungi and bacteria. Our analysis of the culture of $C$. fusiforme and the broth without inoculation showed nearly no correlation in their chemical composition and there are just hydroxymethylfurfurole and benzeneacetic acid in common. From this, we conclude that the fungus could consume and transfer the composition of the culture medium to produce versatile metabolites.

\section{Antifungal activity}

The antifungal activity of the ether extracts from $S$. verrucosa and $C$. fusiforme against the microorganisms considered in the present study was quantitatively assessed (Table 3). 
Table 3. Antimicrobial activity of the ether extract of $S$. verrucosa and C. fusiforme.

\begin{tabular}{lccl}
\hline \multicolumn{4}{c}{$\mathrm{IC}_{80}(\mu \mathrm{g} / \mathrm{mL})$} \\
\hline Test fungi & S. verrucosa & C. fusiforme & $\mathrm{KCZ}^{\mathrm{a}}$ \\
Candida albicans ATCC76615 & 32 & 32 & 0.0625 \\
Cryptococcus neoformans ATCC32609 & 64 & 32 & 0.0625 \\
Trichophyton rubrum & 64 & $\mathrm{ND}^{\mathrm{b}}$ & 0.25 \\
Aspergillus fumigatus & 8 & 32 & 1 \\
Pycricularia oryzae & $>128$ & 128 & 8 \\
\hline
\end{tabular}

${ }^{a}$ Ketoconazole as positive control

${ }^{\mathrm{b}}$ Not determined.

The plant extract displayed broader antimicrobial spectrum and stronger toxicity to most of the tested microbes. But the extract of this endophytic fungus cultures was more toxic to the growth of Cryptococcus neoformans.

\section{Antitumor activity}

The screening of antitumor activity was also conducted, and the results are represented in Table 4 .

Table 4. Antitumor activity of the ether extract of $S$. verrucosa and C. fusiforme.

\begin{tabular}{lrcl}
\hline \multicolumn{3}{c}{$\mathrm{IC}_{50}(\mu \mathrm{g} / \mathrm{mL})$} \\
\hline Cell line & S.vercossa & C. fusiforme & DOX $^{\mathrm{a}}$ \\
A549 & $>100$ & $>100$ & 0.0207 \\
LOVO & $>100$ & 9.11 & 0.734 \\
HL-60 & 42.92 & 4.06 & 0.00190 \\
QGY & 90.78 & 31.23 & 0.0110 \\
\hline
\end{tabular}

${ }^{\mathrm{a}}$ Doxorubicin as positive control

The host plant was moderately inhibitory to all test tumor cells when compared with DOX. The fungus isolated from $S$. verrucosa possessed much better cytotoxic activities than the host plant with most of $\mathrm{IC}_{50}$ values being below $50 \mu \mathrm{g} / \mathrm{mL}$.

Many antibiotics have encountered drug resistance or caused severe adverse drug reactions, and there is an urgent need to search for new antibiotics. Several recent studies have reported the isolation of novel compounds with antimicrobial activity from the cultured endophytic fungi [29-30]. Others have also reported the isolation of endophytic fungi with anti-microbial, anti-cancer and anti-malarial activities from Thai medicinal plants [31-34].

The present study shows that endophytic fungi isolated from S.verrucosa also have a wide range of antifungal and antitumor activities, which were not strong, but better than the host plant, although the vast majority of their chemical constituents were not the same. Interestingly we have hereby disclosed a microbial source of the important phytochemical succedaneum. 
Thus, a smart manipulation of the pathway would lead to a scaled-up fermentation production of some important bioactive compounds. This seems more workable than the desired microbial production of taxol, which is still out of sight although, its producing endophyte has been reported a decade ago [21]. In conclusion, the endophytic fungus of $S$. verrucosa is a versatile producer of the variously bioactive metabolites.

Because of the diversity and complexity of the natural mixtures of bioactive compounds in the crude plant extract and fungal cultures, it is rather difficult to characterize every compound present and elucidate its structure in a single study. According to the information presented, the compounds considered characteristic of $C$. fusiforme are produced by the microorganisms, at least in the selected culture medium. If cultured in other conditions, such as different cultivation time and water content, very different compounds could be produced. However, further investigation is still needed to discover the unidentified/ unknown bioactive constituents in the endophytic fungal isolates and its host.

\section{Experimental}

Plant Material and Prepare for the extract

Samples of S. verrucosa were collected from Yandang Mountain, Zhejiang Province, P.R. China, in November, 2006. Taxonomic identification [35] was performed by Professor Caotong from Shanghai Normal University, Shanghai, China. Voucher specimens of $S$. verrucosa have been deposited at the Herbarium of Department of Pharmacognosy, Second Military Medical University, Shanghai, P.R. China. Plant material was air-dried in the shade prior to extraction. The samples $(10 \mathrm{~g})$ were soaked with diethyl ether $(100 \mathrm{~mL})$ at room temperature. After $24 \mathrm{~h}$, the mixture was filtered through Whatman filter paper using a Buchner funnel. The solvent was removed with a rotary vacuum evaporator at $40^{\circ} \mathrm{C}$. This procedure was repeated three times. The volatile oil yield was $0.6 \%$ and it was stored in dark vials at $4^{\circ} \mathrm{C}$ before analyses [percentage extract yield $(\mathrm{w} / \mathrm{w})$ was estimated as extract weight/starting material weight $\mathrm{x} 100]$.

\section{Isolation of strains}

The endophytic fungal strain 37 was separated from the $S$. verrucosa according to the procedure described by Schulz et al. [36]. Specifically, S. verrucosa was washed with distilled water, sterilized with $75 \%$ ethanol for $1 \mathrm{~min}$ and $2.5 \%$ sodium hypochlorite for $15 \mathrm{~min}$, then rinsed in sterile water for three times. Both borders of sterilized segments were cut off, and the rest was incubated at $28 \pm 1{ }^{\circ} \mathrm{C}$ on PDA medium supplemented with ampicillin $(200 \mu \mathrm{g} / \mathrm{mL})$ and streptomycin $(200 \mu \mathrm{g} / \mathrm{mL})$ to inhibit the bacterial growth until the mycelium or colony originated from the injury surface. The mycelium was purified and cultured under the same conditions. Another segment of the same origin without surface sterilization was cultured as a negative control to check the presence of contaminated microbes on the segment surface. The purified endophytic fungi were numbered and transferred to fresh PDA (Potato Dextrose Agar) slants separately and were kept at $4{ }^{\circ} \mathrm{C}$ after being cultured at $28 \pm 1{ }^{\circ} \mathrm{C}$ for 7 days. 


\section{Fermentation and extraction of the endophytic fungus}

The inoculum was prepared by transferring the periphery of 7-day-old Petri dish cultures of the endophyte into $250 \mathrm{~mL}$ flasks containing broth $\left(100 \mathrm{~mL}\right.$; potato: $200 \mathrm{~g}$, dextrose: $20 \mathrm{~g}, \mathrm{H}_{2} \mathrm{O}: 1000$ $\mathrm{mL})$, followed by shaking $(280 \mathrm{rpm})$ continuously for 7 days at $28 \pm 1{ }^{\circ} \mathrm{C}$. The ferment broth of the endophyte was filtered. The filtrate was extracted with diethyl ether and the obtained extracts were concentrated in a rotatory evaporator at $45{ }^{\circ} \mathrm{C}$. In order to ascertain whether any of the isolates obtained in this study was the constituent of the PDB extract in the substrate, the EtOAc extract of the sterile medium treated equally but without inoculation of the microorganism was subjected to an GCMS comparison showing that all the isolates were indeed produced by the title endophytic fungus.

\section{Essential oil analysis}

Chromatography was performed on a DB-Wax capillary column (30 m x $0.25 \mathrm{~mm}$ ID and $0.25 \mu \mathrm{m}$ film thickness). The electron impact technique $(70 \mathrm{eV})$ was used. The carrier gas was helium at a flow rate of $1.0 \mathrm{~mL} / \mathrm{min}$, and $1 \mu \mathrm{L}$ of sample was injected. The injector and detector temperatures were 230 ${ }^{\circ} \mathrm{C}$ and $200{ }^{\circ} \mathrm{C}$ respectively. The column oven was programmed as follows: initial temperature: $60{ }^{\circ} \mathrm{C}$; initial time $2.0 \mathrm{~min}$; program rate $10{ }^{\circ} \mathrm{C} / \mathrm{min}$; final temperature $250{ }^{\circ} \mathrm{C}$; final time $10 \mathrm{~min}$. The sample was dissolved in $\mathrm{CH}_{2} \mathrm{Cl}_{2}$ and a split injection technique was used. The identification of the compounds was based on comparison of their retention indexes (RI), obtained using n-alkanes (C11-C31), and retention time. They were also confirmed by comparison of their mass spectra with the NIST/NBSWiley library spectra and literature data. Relative percentage amounts were calculated from TIC by the computer.

\section{Identification of the endophyte}

The endophytic fungal strain was identified by the morphological method. The morphological examination was performed by scrutinizing the fungal culture, the mechanism of spore production, and the characteristics of the spores. All experiments and observations were repeated at least twice. The strain was identified as $C$. fusiforme by the Institute of Microbiology, Chinese Academy of Sciences, Beijing, China.

\section{Antifungal activity test}

The essential oil of the host and its endophyte were screened for antifungal activity against Candida albicans, Cryptococcus neoformans, Trichophyton rubrum, Aspergillus fumigatus, Pycricularia oryzae. $\mathrm{IC}_{80}$ were determined using the modified liquid dilution method performed in 96 well microtrays. The antifungal activities of the essential oil are reported in Table 3 and the values presented are an average of triplicate. Sabouraud Dextrose Agar was employed for fungal growth. Dilutions of $S$. Verrucosa and its cultured endophyte extract were prepared in dimethyl sulfoxide (DMSO). The extract solutions were serially diluted (4:1) in 96-well plates. Organisms at a concentration of approximately $1-5 \times 10^{3}$ colony forming units $(\mathrm{CFU} / \mathrm{ml})$ were then added to each well. Plates were 
made in triplicate and incubated at $35^{\circ} \mathrm{C}$ for about $24 \mathrm{~h}$ for Monilia, about $72 \mathrm{~h}$ for Cryptococcus and about $168 \mathrm{~h}$ for hyphomycete and their turbidity obtained by measuring optical density at $630 \mathrm{~nm}$. Test substance concentrations at which fungus proliferation was reduced by $80 \%$ are given as $\mathrm{IC}_{80}$ values. The standard antifungal agent ketoconazole was used as a positive control and experiments were repeated at least three times.

\section{Antitumor activity test}

Test cells were grown in RPMI 1640 including 100 units $/ \mathrm{mL}$ penicillin and $100 \mu \mathrm{g} / \mathrm{ml}$ streptomycin supplemented with $15 \%$ new-born bovine serum (NBS) at $37{ }^{\circ} \mathrm{C}$ in a $5 \% \mathrm{CO}_{2}$ atmosphere. For experimentation, the exponentially growing cells $\left(4 \sim 6 \times 10^{4}\right)$ were used. Then, cells were incubated in the presence of $1000 \mu \mathrm{g} / \mathrm{mL}$ samples in DMSO for $72 \mathrm{~h}$ at $37^{\circ} \mathrm{C}$. After removal of the sample solution and washing with phosphate- buffered saline (pH7.4), $10 \mu \mathrm{l} /$ well of $0.5 \%$ 3-(4,5-dimethyl-2-thiazolyl)2,5-diphenyl-2H-tetrazolium bromide cells (MTT) phosphate-buffered saline solution was added. After a further $4 \mathrm{~h}$ of incubation, $0.04 \mathrm{M} \mathrm{HCl}$ was added. Viable cells were determined by measuring the absorbance at $570 \mathrm{~nm}$. Measurements were performed 3 times, and the concentration required for a $50 \%$ inhibition of viability $\left(\mathrm{IC}_{50}\right)$ was determined graphically.

\section{Acknowledgements}

This work was financially supported by Natural Science Foundation of Fujian Province (No.2008J0097), as well as postdoctoral foundation in Second Military Medical University, Shanghai, P.R. China. We are indebted to Benrong Zuo (Shanghai Normal University, Shanghai, P.R. China) and Linghe Li (Fuzhou University, Fuzhou, P.R. China) for technical assistance.

\section{References}

1. Frahm, J.P.; Kirchhoff, K. Antifeeding effects of bryophyte extracts from Neckera crispa and Porella obtusata against slug Arion lusitanicus. Cryptogamie, Bryologie 2002, 23, 271-275.

2. Asakawa, Y. Biologically active substances found in Hepaticae. In Studies in Natural Products Chemistry; Atta-ur-Rahman, Ed.; Elsevier: Amsterdam, 1988; Vol. 2, pp. 277-292.

3. Asakawa, Y. Biologically active substances from bryophytes. In Bryophytes Development: Physiology and Biochemistry; Chopra, R.N., Bhatla, S.C., Eds.; CRC Press: Boca Raton, 1990, pp. 259-287.

4. Asakawa, Y. Terpenoids and aromatic compounds with pharmacological activity from bryophytes. In Bryophytes: Their Chemistry and Chemical Taxonomy; Zinsmeister, H.D., Mues, R., Eds.; Clarendon Press: Oxford, 1990; pp. 369-410.

5. Asakawa, Y. Chemical constituents of the bryophytes. In Progress in the Chemistry of Organic Natural Products; Herz, W., Kirby, W.B., Moore, R.E., Steglich, W., Tamm, C., Eds.; Springer: Vienna, 1995; Vol. 65, pp. 1-618. 
6. Nagashima, F.; Nondo, M.; Uematsu, T.; Nishiyama, A.; Sato, S.; Sato, M.; Asakawa, Y. Cytotoxic and apoptosis-inducing ent-kaurane-type diterpenoids from the Japanese liverwort Jungermannia truncata Nees. Chem. Pharm. Bull. 2002, 50, 808-813.

7. Asakawa, Y. Chemical constituents of the Hepaticae. In Progress in the Chemistry of Organic Natural Products; Herz, W., Grisebach, H., Kirby, G.W., Eds.; Springer: Vienna, 1982; Vol. 42, pp. 1-285.

8. Asakawa, Y. Phytochemistry of Bryophytes. Biologically Active Terpenoids and Aromatic Compounds from Liverworts. In Phytochemicals in Human Health Protection, Nutrition, and Defense; Romeo, J.T., Ed.; Kluwer Academic/Plenum: New York, 1999; pp. 319-342.

9. Lars, S.; Ana, S.; Mariana, S. Rarity patterns in members of the Lophoziaceae/Scapaniaceae complex occurring North of the Tropics - Implications for conservation. Biol. Conserv. 2007, 352-359.

10. Yoshida, T.; Toyota, M.; Asakawa, Y. Scapaundulins A and B, two novel dimeric labdane diterpenoids, and related compounds from the Japanese liverwort Scapania undulata (L) Dum. Tetrahedron Lett. 1997, 38, 1975-1978.

11. Adioa, A.M.; Paulb, C.; Klotha, P.; Königa, W.A. Sesquiterpenes of the liverwort Scapania undulata. Phytochemistry 2004, 65, 199-206.

12. Nagashima, F.; Asakawa, Y. Sesqui- and diterpenoids from two Japanese and three European liverworts. Phytochemistry 2001, 56, 347-352.

13. Tazakia, H.; Hayashida, T.; Furuki, T.; Nabeta, K. Terpenoid from the liverwort Scapania bolandeli. Phytochemistry 1999, 52, 1551-1553.

14. Geis, W.; Buschauer, B.; Becker H. cis-Clerodandes from axenic cultures of the liverwort Scapania nemorea. Phytochemistry 1999, 51, 643-649.

15. Mues, R.; Huneck, S.; Connolly. J.D.; Rycroft, D.S. Scapaniapyrone-A, a novel aromatic constituent of the liverwort Scapania undulata. Tetrahedron Lett. 1988, 29, 6793-6796.

16. Stone, J.K.; Bacon, C.W.; White, J.F. In An Overview of Endophytic Microbes: Endophytism Defined in Microbial Endophytes; Bacon, C.W., White, J.F., Eds.; Marcel Deker: New York, 2000.

17. Faeth, S.H.; Hammon, K.E. Fungal endophytes in oak trees: long-term patterns of abundance and association with leafminers. Ecology 1997, 78, 810-819.

18. Huang, W.Y.; Cai, Y.Z.; Xing, J.; Corke, H.; Sun, M. Potential antioxidant resource: endophytic fungi isolated from traditional Chinese medicinal plants. Econ Bot. 2007, 61, 14-30.

19. Fisher, P.J.; Anson, A.E.; Petrini, O. Antibiotic activity of some endophytic fungi from ericaceous plants. Bot. Helv. 1984, 94, 249-253.

20. Gurney, K.A.; Mantle, P.G. Biosynthesis of 1-N-methylalbonoursin by an endophytic Streptomyces sp. isolated from perennial ryegrass. J. Nat. Prod. (Lloydia) 1993, 56, 1194-1198.

21. Stierle, A.; Strobel, G.; Stierle, D. Taxol and taxane production by Taxomyces andreanae, an endophytic fungus of Pacific Yew. Science 1993, 260, 214-216.

22. Tan, R.X.; Zou, W.X. Endophytes: a rich source of functional metabolites. Nat. Prod. Rep. 2001, $18,448-459$.

23. Hawksworth, D.C; Rossman, A.Y. Where are the undescribed fungi. Phytopathology 1987, 87 888-891. 
24. Nagashima, F.; Suda, K.; Asakawa, Y. Cadinane-type sesquiterpenoids from the liverwort Scapania undulata. Phytochemistry 1994, 37, 1323-1325.

25. Asakawa, Y.; Inoue, H.; Toyota, M.; Takemoto, T. Sesqui- terpenoids of fourteen Plagiochila species. Phytochemistry 1980, 19, 2623-2626.

26. Ohta, Y.; Andersen, N.H.; Liu, C.B. Sesquiterpene con-stituents of two liverworts of genus Diplophyllum, Novel eudesmanolides and cytotoxicity studies for enantiomeric methylene lactones. Tetrahedron 1977, 33, 617-628.

27. Matsuo, A.; Atsumi, K.; Nakayama, M. Isolation of seven verrucosane diterpenoids from the liverwort Scapania bolanderi. Z. Naturforsch. 1984, 39b, 1281-1285.

28. Asakawa, Y. Chemosystematics of the Hepaticae. Phytochemistry 2004, 65, 623-669.

29. Liu, J.Y.; Liu, C.H.; Zou, W.X.; Tan, R.X. Leptosphaeric acid, a metabolite with a novel skeleton from Leptosphaeria sp. IV403, an endophytic fungus in Artemisia annua. Helv. Chim. Acta 2003, 86, 657-660.

30. Schulz, B.; Boyle, C.; Draeger, S.; Römmert, A.K.; Krohn, K. Endophytic fungi: a source of novel biologically active secondary metabolites. Mycol. Res. 2002, 106, 996-1004.

31. Liu, C.H.; Zou, W.X.; Lu, H.; Tan, R.X. Antifungal activity of Artemisia annua endophyte cultures against some phytopathogenic fungi. J. Biotechnol. 2001, 88, 277-283.

32. Liu, C.H.; Liu, J.Y.; Huang, L.L.; Zou, W.X.; Tan, R.X. Absolute configuration of keisslone, a new antimicrobial metabolite from Keissleriella sp. YS4108, a marine filamentous fungus. Planta Med. 2003, 69, 481-483.

33. Gary, A.S. Endophytes as sources of bioactive products. Microbes Infect. 2003, 5, 535-544.

34. Wiyakrutta, S.; Sriubolmas, N.; Panphut, W.; Thongon, N. Endophytic fungi with anti-microbial, anti-cancer and anti-malarial activities isolated from Thai medicinal plants. World J. Microbiol. Biotechnol. 2004, 20, 265-272.

35. Gao, Q.; Cao, T. Bryophyta: Hepaticae, Anthocerotae. In Flora Yunnanica; eds.; Science Press: Beijing, 2000; Vol. 17, p. 247.

36. Schulz, B.; Sucker, J.; Aust, H.J.; Krohn, K.; Ludewig, K.; Jones, P.G.; Doring, D. Biologically active secondary metabolites of endophytic Pezicula species. Mycol. Res. 1995, 99, 1007-1015.

Sample Availability: Available from the authors.

(C) 2008 by the authors; licensee Molecular Diversity Preservation International, Basel, Switzerland. This article is an open-access article distributed under the terms and conditions of the Creative Commons Attribution license (http://creativecommons.org/licenses/by/3.0/). 